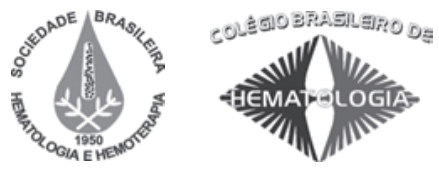

\title{
Uso da fluorescência de raios $X$ portátil (XRF) in vivo como técnica alternativa para acompanhamento dos níveis de ferro em pacientes com sobrecarga de ferro The in vivo use of portable $X$-ray fluorescence as an alternative technique for the accompaniment of iron levels in patients with iron loading
}

Marcelo Estevam ${ }^{1}$

Carlos R. Appoloni ${ }^{1}$
Foi investigada a viabilidade da aplicação da técnica de fluorescência de raios $X$ (XRF) como alternativa para acompanhamento dos níveis de ferro em pacientes portadores de talassemia maior (beta-thalassemia) e hemocromatose hereditária $(\mathrm{HH})$. As medidas foram realizadas no Hemocentro do Hospital Universitário e no Laboratório de Física Nuclear Aplicada da Universidade Estadual de Londrina. Foi acompanhada uma portadora de talassemia maior e quatro pessoas sadias. Foi utilizado um sistema portátil de XRF constituído de uma fonte de Raios X de 238Pu e um detector de SiPIN diodo, para as medidas in vivo na mão. O sistema foi calibrado medindo simulados de solução aquosa com 15 a 150 ppm de ferro. A duração de cada medida foi de $50 \mathrm{~s}$. O limite de detecção (LLD) atingido foi de 13 ppm de ferro. A dose de radiação na pele foi de $10 \mathrm{mSv}$. A paciente de talassemia apresentou $74 \pm$ 6 ppm de ferro, enquanto pessoas sadias apresentaram valor médio de $53 \pm 5$ ppm de ferro. Os resultados estão de acordo com a literatura, que informa níveis de ferro na pele de 15 a 60 ppm em pessoas sadias e de 70 a 150 ppm em pacientes portadores da talassemia maior. Foi concluído ser viável a aplicação da XRF para acompanhamento de pacientes de talassemia maior e HH. Rev. Bras. Hematol. Hemoter. 2009;31(3):153-159.

Palavras-chave: Talassemia; sobrecarga de ferro; fluorescência.

\section{Introdução}

Portadores de talassemia maior (beta-thalassemia) ou hemocromatose hereditária $(\mathrm{HH})$ apresentam um elevado acúmulo de ferro no organismo devido ao tratamento da doença e à própria doença, respectivamente. Atualmente, o monitoramento do acúmulo de ferro é realizado através do acompanhamento da ferritina sérica.

Alguns estudos informam que a sobrecarga férrea do organismo é proporcional à concentração de ferro na pele. ${ }^{1,2,3}$ Apresentamos neste trabalho medidas in vivo na pele da mão de quatro pessoas sadias e um portador de talassemia maior através da técnica de fluorescência de raios X (XRF), técnica precisa, rápida, não invasiva e capaz de repetições regulares.

O objetivo desse trabalho é investigar os resultados das medidas de ferro em pele humana utilizando um aparato experimental portátil de XRF, verificando se estes níveis são compatíveis com os níveis associados à doença talassemia maior e HH.

${ }^{1}$ Professor. Universidade Estadual de Londrina, CCE, Depto. de Física, Laboratório de Física Nuclear Aplicada, Londrina-PR.

Universidade Estadual de Londrina, CCE, Depto. de Física, Laboratório de Física Nuclear Aplicada, Londrina-PR.

Correspondência: Marcelo Estevam

Universidade Estadual de Londrina Depto. de Física

Rodovia Celso Garcia Cid, Pr 445 Km 380

86051-990 - Londrina-PR - Brasil

Caixa Postal 6001

Fax: (55 43) 3371-4166

E-mail: marceloestevam@yahoo.com.br - appoloni@uel.br

Doi: 10.1590/S1516-84842009005000043 
Tabela 1. Resumo dos artigos que reportam resultados de XRF in vivo

\begin{tabular}{|c|c|c|c|c|c|c|}
\hline Autor / ano & $\begin{array}{l}\text { Técnicas } \\
\text { de XRF }\end{array}$ & Fonte de Excitação & Matriz / amostra & $\begin{array}{l}\text { Tempo de } \\
\text { Medidas }\end{array}$ & Dose (Local) & $\begin{array}{c}\text { Elementos e } \\
\text { Limite de detecção (LLD) }\end{array}$ \\
\hline $\begin{array}{c}\text { D. S. MacLean et al } \\
1995^{4}\end{array}$ & XRF & ${ }^{241} \mathrm{Am}(500 \mathrm{mCi})$ & $\begin{array}{l}\text { Fêmeas de ratos / } \\
\text { pele }\end{array}$ & $10 \mathrm{~min}$ & 0.68-1.1 rem & I (não informado) \\
\hline $\begin{array}{c}\text { A. Shukri et al } \\
1995^{5}\end{array}$ & KXRF & ${ }^{109} \mathrm{Cd}(100 \mathrm{mCi})$ & $\begin{array}{l}\text { Simulados de } \\
\text { FeSO4 }\end{array}$ & $20 \mathrm{~min}$ & $5 \mathrm{mSv}$ & $\mathrm{Fe}(119 \mathrm{ppm})$ \\
\hline $\begin{array}{c}\text { D. S. MacLean et al } \\
1996^{6}\end{array}$ & XRF & ${ }^{241} \mathrm{Am}(500 \mathrm{mCi})$ & Humanos / pele & $25 \min$ & 242 mrem & I (não informado) \\
\hline $\begin{array}{c}\text { J. M. O'Meara et al } \\
1998^{7}\end{array}$ & XRF & $57 \mathrm{Co}(1 \mathrm{mCi})$ & $\begin{array}{c}\text { Simulados / } \\
\text { gesso de Paris }\end{array}$ & $60 \mathrm{~min}$ & $80 \mathrm{nSv}$ & $\mathrm{U}(20 \mathrm{ppm})$ \\
\hline $\begin{array}{l}\text { J. M. O'Meara et al } \\
2000^{8}\end{array}$ & $\begin{array}{l}\text { Polarizado } \\
\text { XRF }\end{array}$ & $\begin{array}{l}\text { Tubo de Raios } X \\
(250 \mathrm{kV} \text { e } 15 \mathrm{~mA})\end{array}$ & $\begin{array}{c}\text { Simulados / } \\
\text { soluções aquosas } \\
\text { dopadas de } \mathrm{Hg}\end{array}$ & $\begin{array}{c}\text { Não } \\
\text { informado }\end{array}$ & $\begin{array}{l}\text { 8-14 mGy/h } \\
\text { (pele) }\end{array}$ & $\mathrm{Hg}(26-54 \mathrm{ppm})$ \\
\hline $\begin{array}{l}\text { D. A. Bradley et al } \\
2000^{9}\end{array}$ & XRF & $\begin{array}{r}\text { Tubo de Raios } X \\
(15 \mathrm{kV}, 23 \mathrm{~mA})\end{array}$ & $\begin{array}{c}\text { Simulados / } \\
\text { soluções aquosas } \\
\text { dopadas de } \\
\mathrm{Fe}, \mathrm{Zn} \text { e } \mathrm{Cu}\end{array}$ & $33.3 \mathrm{~min}$ & $16 \mathrm{mSv}$ & $\begin{array}{c}\mathrm{Fe}(20 \mathrm{ppm}), \mathrm{Zn}(10 \mathrm{ppm}) \\
\text { e Cu (5 ppm) }\end{array}$ \\
\hline $\begin{array}{l}\text { J. M. O'Meara } \\
2004^{10}\end{array}$ & XRF & $\begin{array}{l}\text { Tubo de Raios X } \\
(200 \text { kV e } 20 \text { mA) }\end{array}$ & Humanos / rim & $50 \mathrm{~min}$ & $5 \mathrm{mSv}$ & $\mathrm{Hg}(19 \mathrm{ppm})$ \\
\hline $\begin{array}{l}\text { P. A. Ali } \\
1998^{11}\end{array}$ & $\begin{array}{l}\text { Polarizado } \\
\text { XRF }\end{array}$ & $\begin{array}{l}\text { Tubo de Raios } X \\
(220 \mathrm{kV})\end{array}$ & $\begin{array}{c}\text { Humanos / cabeça } \\
\text { e tumores de } \\
\text { pescoço }\end{array}$ & $33.3 \mathrm{~min}$ & 3 mGy & $\mathrm{Pt}(5,6 \mathrm{ppm})$ \\
\hline $\begin{array}{c}\text { J. Shakeshaft } \\
1993^{12}\end{array}$ & XRF & ${ }^{153} \mathrm{Gd}(24 \mathrm{GBq})$ & Humanos / rim & $30 \mathrm{~min}$ & 100 Sv (rim) & Au (30-60 ppm) \\
\hline $\begin{array}{l}\text { J. Börjesson } \\
1995^{13}\end{array}$ & XRF & Tubo de Raios $X$ & Humanos / rim & $30-50 \mathrm{~min}$ & 5 mGy (pele) & $\mathrm{Hg}(12-45 \mathrm{ppm})$ \\
\hline $\begin{array}{c}\text { J. O. Christoffersson } \\
1983^{14}\end{array}$ & $\begin{array}{l}\text { Polarizado } \\
\text { XRF }\end{array}$ & $\begin{array}{c}\text { Tubo de Raios X } \\
(150 \mathrm{kV}, 15 \mathrm{~mA}) \mathrm{e} \\
241 \mathrm{Am}\end{array}$ & $\begin{array}{l}\text { Humanos / } \\
\text { córtex do rim }\end{array}$ & $30 \mathrm{~min}$ & $\begin{array}{c}10 \mathrm{mGy} \\
\left(5 \mathrm{~cm}^{2} \mathrm{da} \text { pele }\right)\end{array}$ & Cd (8-20 ppm) \\
\hline $\begin{array}{c}\text { J. O. Christoffersson } \\
1987^{15}\end{array}$ & XRF & $\begin{array}{l}\text { Tubo de Raios } X \\
(150 \mathrm{kV}, 15 \mathrm{~mA})\end{array}$ & $\begin{array}{l}\text { Humanos / } \\
\text { córtex do rim }\end{array}$ & $\begin{array}{c}33.3 \mathrm{~min} \\
\text { (tempo vivo) }\end{array}$ & 1,8 mGy (rim) & Cd (8-20 ppm) \\
\hline $\begin{array}{c}\text { F.H.H. Al-Ghorabie et al } \\
2001^{16}\end{array}$ & XRF & ${ }^{99} \mathrm{mTc} \mathrm{e}^{133} \mathrm{Xe}$ & $\begin{array}{l}\text { Simulados / } \\
\text { garrafas de } \\
\text { polietileno } \\
\text { com água }\end{array}$ & $\begin{array}{c}\text { Não } \\
\text { informado }\end{array}$ & 7 mGy (pele) & Pt (não informado) \\
\hline
\end{tabular}

\section{Fluorescência de raios $X$ in vivo}

Na detecção de ferro por XRF devido às energias relativamente baixas dos fótons fluorescentes emitidos pelo ferro de energias $\mathrm{K} \alpha$ e $\mathrm{K} \beta 6,40 \mathrm{keV}$ e 7,06 keV respectivamente, a profundidade da penetração no tecido é pequena, da ordem de alguns milímetros. ${ }^{1,4,5}$ Isto significa que uma medida não invasiva deve ser feita em tecidos superficiais, tais como a pele, e correlacionada então com os resultados de exames rotineiros realizados pelos portadores de talassemia maior.

Técnicas não invasivas para determinação de concentrações de metais pesados in vivo foram desenvolvidas extensivamente durante os últimos 25 anos. A Tabela 1 resume os principais trabalhos relacionados com XRF in vivo. Atual- mente, a técnica de XRF in vivo pode ser utilizada para aplicações biomédicas, pois nas últimas décadas foram desenvolvidos detectores de raios $\mathrm{X}$ resfriados por efeito Peltier, não sendo necessário, para sua operação, nitrogênio líquido, e com resolução em energia suficiente para medidas in vivo, assim como fontes de baixa atividade proporcionando doses seguras de radiação.

Poucos trabalhos estudaram o elemento ferro no organismo humano. A maior parte dos estudos determina os níveis de metais como cádmio, ouro, iodo, chumbo, mercúrio e platina no organismo. Para a detecção desses metais utilizando XRF in vivo é necessário conhecer a localização orgânica da sua concentração. A Tabela 2 mostra a localização dos estudos in vivo de alguns metais no organismo humano, bem como o número atômico desses elementos. ${ }^{17}$ 
Tabela 2. Principal localização das medidas de XRF in vivo e número atômico para alguns elementos

\begin{tabular}{ccc}
\hline Elemento & $\begin{array}{c}\text { Local da medida de } \\
\text { XRF in vivo }\end{array}$ & $\begin{array}{c}\text { Número } \\
\text { atômico }\end{array}$ \\
\hline Cádmio (Cd) & Fígado e rim & 48 \\
Ouro (Au) & Rim, fígado e ossos & 79 \\
lodo (I) & Glândula tireoide & 53 \\
Chumbo $(\mathrm{Pb})$ & $\quad$ Ossos \\
Mercúrio $(\mathrm{Hg})$ & $\begin{array}{c}\text { Ossos, figado, rim e } \\
\text { glândula tireoide } \\
\text { Rlatina }(\mathrm{Pt})\end{array}$ & 82 \\
& $\begin{array}{c}\text { Rim, figado e } \\
\text { neoplasias }\end{array}$ & 78 \\
\hline
\end{tabular}

Em certas condições, podem ser usadas as concentrações de metais em sangue e urina para avaliar a concentração de metal em um órgão interno. Porém, em geral estas relações não são simples ou bem conhecidas. As concentrações de metais em sangue e urina podem ser influenciadas por vários fatores como o nível de exposição contínua, função renal e diferenças individuais na cinética do metal. ${ }^{9}$

Em contraste com métodos in vitro, uma medida in vivo representa uma estimativa direta da concentração de metal apresentada no órgão. As duas principais técnicas in vivo são a análise por ativação neutrônica (NAA), empregada em pesquisas com animais, e fluorescência de raios X (XRF). ${ }^{20}$ A XRF in vivo pode ser usada para quantificar a concentração de ferro, cádmio, mercúrio, chumbo e outros metais pesados diretamente nos órgãos e tecidos do corpo.

\section{Talassemia maior}

O portador da talassemia maior apresenta uma anemia por falta ou má formação da hemoglobina. Transfusões regulares de concentrado de hemácias associadas ao uso de quelantes de ferro aumentaram a sobrevida de pacientes com talassemia maior. O uso da deferroxamina (DFO), introduzida no início da década de 60 , foi aceito como tratamento quelante padrão em pacientes cronicamente transfundidos com sobrecarga de ferro. No entanto, foi aplicada mais de uma década depois, nos países com condições de arcar com os custos relativamente elevados desta forma de terapia. ${ }^{3}$ A comprovação inequívoca do benefício desta modalidade de tratamento veio através de dois estudos clínicos em pacientes com talassemia maior, com mais de dez anos de seguimento, que demonstraram que o uso da DFO está associado a um aumento da sobrevida e menor risco das complicações relacionadas ao ferro. ${ }^{21,22}$

Embora o ferro hepático seja usado como padrão-ouro da avaliação do acúmulo de ferro em pacientes talassêmicos, é no músculo cardíaco que o ferro exerce seu papel mais importante, sendo a doença cardíaca a principal causa de morte nestes pacientes. ${ }^{3,23}$ A quelação com a DFO é capaz não só de mudar as curvas de sobrevida de pacientes talassêmicos por diminuição das mortes por doença cardíaca, mas também de reverter alterações cardíacas já estabelecidas quando usada continuamente por via endovenosa e por períodos superiores a um ano. ${ }^{24,25,26}$

Um dos problemas que motivou o estudo da aplicabilidade da XRF in vivo está relacionado ao manejo da terapia quelante e sua relação com a doença cardíaca e a falta de métodos alternativos de avaliação do ferro cardíaco. Após a demonstração de que os níveis da ferrítina sérica e ferro hepático muitas vezes não se correlacionam com a progressão da doença cardíaca, a busca por estes métodos tornou-se uma nova área de interesse científico. ${ }^{27}$ Recentemente, a utilização de métodos de ressonância magnética (RM) para avaliação do ferro cardíaco e hepático abriu a possibilidade de uma terapia quelante mais racional. No entanto, estes métodos ainda carecem de padronização, o que torna até mesmo a análise de artigos científicos de grupos distintos complicada por diferenças nos protocolos de avaliação. ${ }^{27}$ Nosso grupo trabalha no emprego da XRF in vivo para monitoramento da sobrecarga de ferro em portadores de talassemia maior e hemocromatose hereditária. Esta técnica com recursos tecnológicos atuais, apresenta precisão adequada.

\section{Hemocromatose hereditária $(\mathrm{HH})$}

O termo hemocromatose se refere às doenças nas quais há um aumento progressivo nos estoques corpóreos de ferro, o que ocasiona sua deposição nas células parenquimatosas do coração, hipófise, gônadas, pâncreas, fígado e outros órgãos, com posterior dano estrutural e funcional destes. ${ }^{30} \mathrm{Na}$ ausência de terapêutica adequada e precoce, qual seja a remoção da sobrecarga orgânica de ferro, surgem complicações da doença: insuficiência cardíaca, disfunção hipofisária e gonadal, diabetes mellitus, cirrose hepática e carcinoma hepatocelular, entre outras. ${ }^{28,29,30}$

Em condições normais, o conteúdo total de ferro no organismo é muito estável: cerca de 4 a 5 g. Entretanto, nos pacientes sintomáticos com hemocromatose hereditária, o que geralmente ocorre entre os 40 e 60 anos de idade, os estoques do metal situam-se entre 20 e 40 g, como consequência da hiperabsorção intestinal de ferro, muito acima das necessidades orgânicas, a despeito de ingestão dietética com conteúdo normal do metal. . $^{30-33}$

A remoção de ferro por venissecção ainda é a principal modalidade terapêutica para a $\mathrm{HH}$, com indícios evidentes de melhora da sobrevida em vários estudos. ${ }^{34-37}$ Assim como no tratamento da talassemia, os níveis de ferritina podem oscilar muito durante o tratamento. 


\section{Casuística e Método}

Além dos fatores fundamentais para a eficácia da XRF in vivo, como número atômico dos elementos, energia da radiação empregada, região e profundidade de interação da radiação, o efeito que é mais relevante em aplicações in vivo é a dose de radiação, tendo em vista seus efeitos orgânicos. A equação 1 estima a dose da radiação em relação à distância fonte - amostra, tempo de exposição, fatores geométricos e característica da fonte de $238 \mathrm{Pu} .{ }^{38}$

$$
\mathrm{D}=1,96 \cdot 10^{-7} \cdot \Theta / \mathrm{r}^{2}\left[\mathrm{~S} v \cdot \mathrm{S}^{-1}\right]
$$

onde $r$ é a distância fonte - amostra, e $\Theta$ contém os fatores geométricos.

A profundidade de interação da radiação também foi calculada. O coeficiente de atenuação linear $(\mu)$ da pele para uma energia de 8,4 KeV é 9,036 $\mathrm{cm}^{-1} .{ }^{39}$ A equação da passagem da radiação eletromagnética através da matéria se caracteriza por uma absorção exponencial, equação $2 .^{38}$

$$
\mathrm{I}=I_{0} e^{-\mu x}
$$

Para a linha da fluorescência do ferro, $\mathrm{K} \alpha=6,4 \mathrm{keV}$, decorre que $\mu=20,4 \mathrm{~cm}^{-1}$. Assim $13 \%$ dos fótons de fluorescência da camada $\mathrm{K}$ gerados a uma profundidade de $1 \mathrm{~mm}$ emergem da pele. Portanto a detecção do ferro pelo arranjo experimental utilizado é predominante nas camadas mais superficiais da pele.

É importante ressaltar a aplicabilidade do sistema XRF utilizado por ser pequeno e portátil (PXRF) e operante em temperatura ambiente. Seus resultados mostram-se compatíveis com outras técnicas como scanning electron microscopy (SEM), energy dispersive X-ray fluorescence (EDXRF), electronic paramagnetic resonance (EPR) e Möessbauer spectroscopy. ${ }^{40,41}$

Foi utilizado nesse trabalho um detector de Si-PIN Fotodiodo, resfriado por efeito Peltier. A resolução em energia para a linha de 5,9 eV do Mn é de 220 eV (Figura 1). A fonte de raios $\mathrm{X}$ utilizada foi de ${ }^{238} \mathrm{Pu}$, que apresenta linhas de energia de 13 e $17 \mathrm{keV}$. Os espectros de XRF foram analisados usandose um grupo de programas do ÁXIL - IAEA. ${ }^{40}$

O objetivo de nosso estudo era saber se poderíamos medir uma quantidade significativa de ferro de maneira não invasiva. Foi escolhida para a medida a mão, região extrema do corpo, assim os efeitos da radiação são minimizados e sua imobilização é facilitada, como mostra a Figura 2.

\section{Resultados e Discussão}

Simulados de pele foram confeccionados para o levantamento da curva de sensibilidade ou calibração, apresentada na Figura 3, e posterior quantificação dos níveis de ferro medidos in vivo.

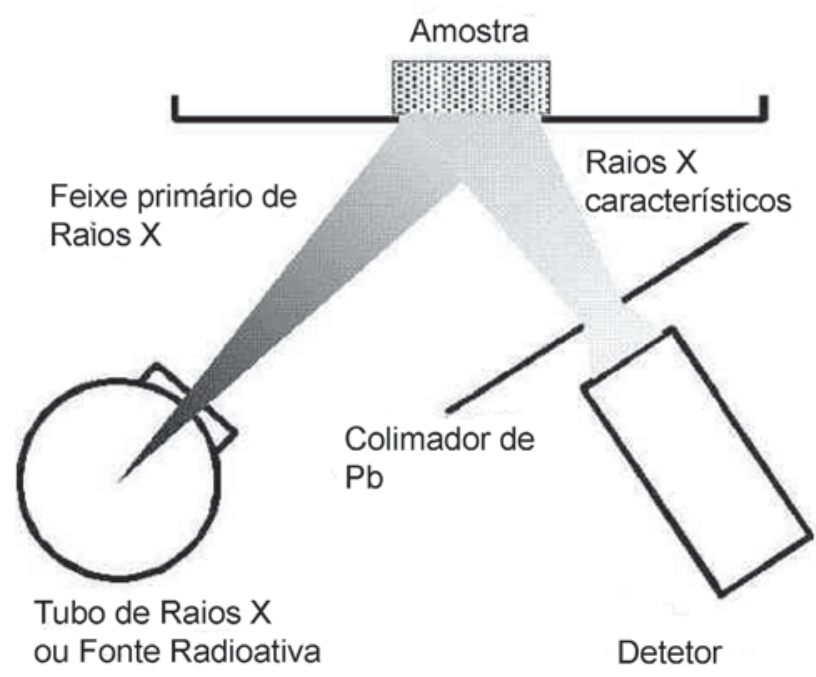

Figura 1. Arranjo experimental da XRF

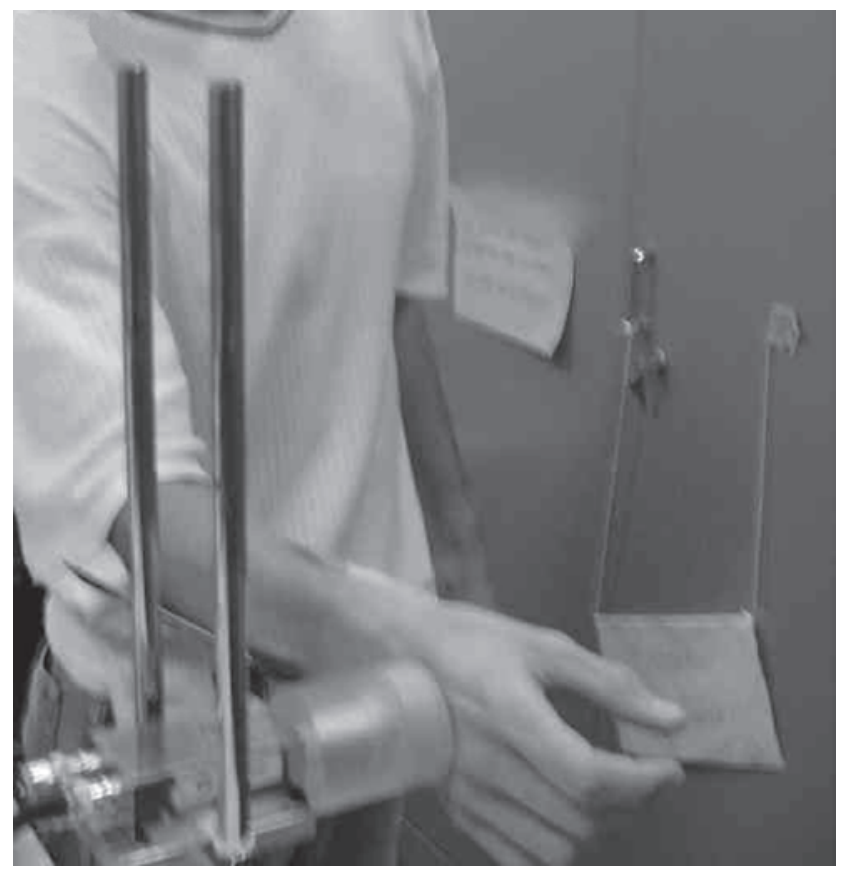

Figura 2. Medida de XRF in vivo na pele da mão

Os simulados foram elaborados com soluções aquosas dopadas com sulfato de ferro $\left(\mathrm{FeSO}_{4} 7 \mathrm{H}_{2} \mathrm{O}\right)$ com concentrações de 20 ppm, 50 ppm, 60 ppm, 100 ppm, 120 ppm, 140 ppm e $160 \mathrm{ppm}$ de ferro. Também foram confeccionados simulados testes para validação do sistema com concentrações de ferro de $30 \mathrm{ppm}$, $70 \mathrm{ppm}$ e $90 \mathrm{ppm}$. A incerteza nos valores dos simulados foi de \pm 5 ppm de ferro devido à instabilidade no equilíbrio químico e erros na confecção. ${ }^{42}$

$\mathrm{O}$ ajuste linear dos pontos da curva de sensibilidade (Figura 3) alcançou um coeficiente de determinação de 0,98, mostrando um ajuste adequado para quantificação das medidas. 


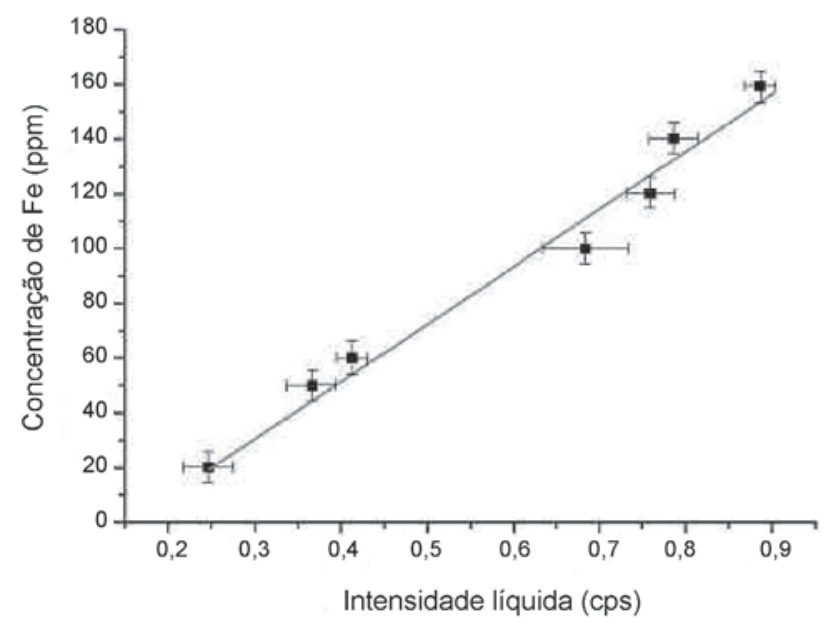

Figura 3. Curva de sensibilidade para o arranjo experimental

Tabela 3. Comparação entre os valores das soluções certificadas e valores medidos com $95 \%$ de confiança

\begin{tabular}{lccc}
\hline $\begin{array}{c}\text { Solução certificada } \\
\text { (ppm de ferro) }\end{array}$ & $30 \pm 5$ & $70 \pm 5$ & $90 \pm 5$ \\
$\begin{array}{l}\text { Valor medido } \\
\text { (ppm de ferro) }\end{array}$ & $37 \pm 6$ & $76 \pm 4$ & $92 \pm 6$ \\
\hline
\end{tabular}

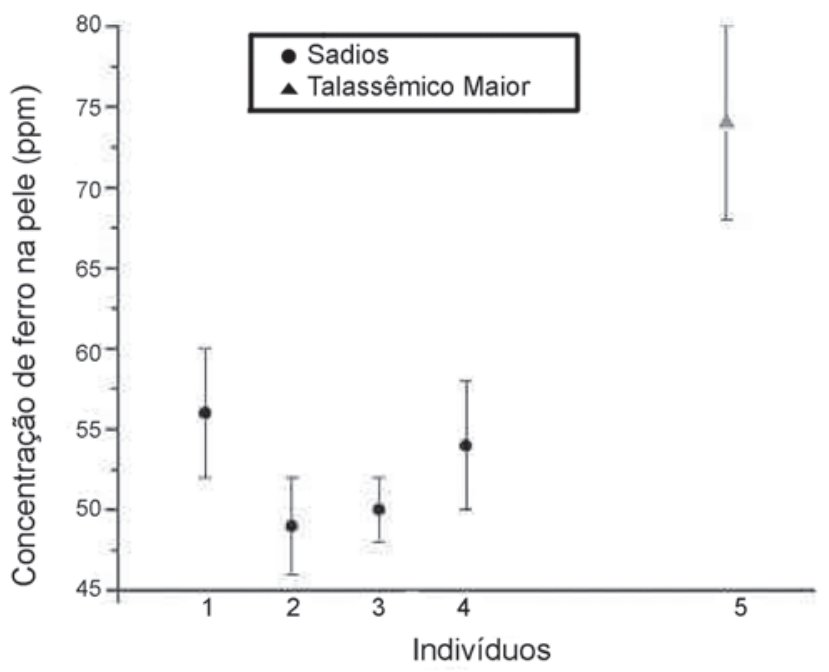

Figura 4. Níveis ferro na pele de sadios e de portadores de talassemia maior medidos por XRF in vivo

A Tabela 3 mostra a validação do sistema, comparando os valores das soluções certificadas com os valores medidos com $95 \%$ de confiança.

Posteriormente, foram relacionados os níveis de ferro de pessoas sadias com um portador de talassemia maior, na época da medida, uma menina com 7 anos de idade.
Todos os valores apresentados na Tabela 3 estão dentro das incertezas validando assim a metodologia utilizada.

Medidas nos simulados de pele forneceram um limite mínimo de detecção (LLD) de 13 ppm para o ferro, usandose períodos de medida de 50 segundos e dose na superfície da pele de $3 \mathrm{mSv}$, para medidas realizadas em triplicata, resultando em uma dose total menor que $10 \mathrm{mSv}$.

O limite de deteç̧ão foi compatível para medidas confiáveis comparadas com os níveis de ferro informados pela literatura, de 15 a $60 \mathrm{ppm}$ em pessoas sadias e de 70 a 150 ppm em pacientes portadores da talassemia maior. ${ }^{1}$

Os valores das intensidades líquidas de ferro para os quatro humanos sadios e para a portadora de talassemia maior foram quantificados através da curva de sensibilidade, obtendo-se os valores apresentados na Figura 4.

Durante o tratamento de transfusões regulares e uso da DFO como tratamento quelante padrão em pacientes cronicamente transfundidos com sobrecarga de ferro, ${ }^{3}$ caso a quelação não seja eficiente, será observada uma elevação nos níveis de ferrítina sérica.

\section{Conclusão}

Através do estudo apresentado concluímos que esse aumento pode ser monitorado através da XRF como demonstra a Figura 4. Portanto, concluímos como viável a utilização da XRF in vivo para medidas da concentração de ferro na pele nos níveis relacionados a humanos sadios e talassêmicos.

$\mathrm{O}$ aumento de ferro na pele nos leva a crer que o tratamento da talassemia maior causa um aumento dos níveis de ferro no corpo todo, inclusive na pele. Foi possível quantificar níveis de ferro medido em pessoas sadias com $53 \pm 5$ ppm e o nível de ferro da portadora de talassemia maior com $74 \pm 6$ ppm, uma diferença estatisticamente confiável.

Atualmente nossos trabalhos estão voltados para a correlação dos níveis de ferro apresentados na pele com níveis em órgãos como o fígado e coração, medidos por biópsia, assim como com os níveis de ferro obtidos através de exames de ferritina sérica, ferro sérico, transferrina, índice de saturação e capacidade total de ligação de ferro. Enfim, visa-se utilizar a XRF in vivo, num futuro próximo, como meio alternativo e não invasivo para o monitoramento da eficácia do tratamento de quelação em pacientes de talassemia maior e hemocromatose hereditária.

\section{Agradecimentos}

A todos que se submeteram voluntariamente às medidas de XRF in vivo, ao Prof. Dr. Fábio Luis Melquiades e Prof. Dr. Paulo Sérgio Parreira pelo suporte técnico. À Dra. Cristina Faune, do Hemocentro do Hospital Universitário da UEL, pelo apoio. 


\section{Abstract}

The viability of the $X$-ray fluorescence technique as an alternative to follow up iron levels in patients suffering from thalassemia major (beta-thalassemia) and hereditary hemochromatosis was investigated. The measurements were carried out in the University Hospital Blood Center and in the Laboratory of Applied Nuclear Physics of the State University of Londrina. One thalassemia major patient and four healthy individuals were enrolled in the study. A portable $X$-ray fluorescence system consisting in a $238 \mathrm{Pu} X$-ray source and a Si PIN diode detector was used for in vivo measurements of the hand. The system was calibrated using 15 to 150 ppm concentrations of iron in an aqueous solution. The duration of each measurement was 50 seconds. The achieved detection limit was 13 ppm of iron. The radiation dose on the skin was $10 \mathrm{mSv}$. The thalassemia patient presented with $74 \pm 6 \mathrm{ppm}$ of iron, whereas the healthy individuals presented with an average of $53 \pm 5$ ppm of iron. The results are in accordance with prior publications that reported iron levels in the skin of between 15 and 60 ppm in healthy people and between 70 and 150 ppm in thalassemia major patients. In conclusion the $X$-ray Fluorescence technique is viable in the follow-up of thalassemia major and hereditary hemochromatosis patients. Rev. Bras. Hematol. Hemoter. 2009;31(3):153-159.

Key words: Thalassemia; iron-loading; fluorescence.

\section{Referências Bibliográficas}

1. Gorodetsky R, Lowenthal E, Goldfarb A, Rachmilewitz EA. Evaluation of iron load and clearance in patients with betathalassemia. Ann. NY Acad. Sci. 1990;(612):568-72.

2. Estevam M, Malvezi AD, Borges CL, Pinge-Filho P, Appoloni CR. Evaluation of Fe employing X-Ray Fluorescence Methodology (XRF) in mice skin during acute phase of experimental infection with Trypanosoma cruzi. VI Latin American Symposium on Nuclear Physics and Applications. AIP Conf. Proc. 2007; (884): 494-6.

3. Olivieri NF, Brittenham GM. Iron-chelating therapy and the treatment of thalassemia. Blood. 1997;89(3):739-61.

4. MacLean DS, Robertson JD, Jay M, Stalker DJ. Noninvasive measurement of protein release from subcutaneous depo formulations in vivo using X-ray fluorescence. J Control Release. 1995;(34):167-73.

5. Shukri A, Green S, Bradley DA. A feasibility study: in vivo x-ray fluorescence of iron using 109Cd. Appl Radiat Isot. 1995;46 (6-7):625.

6. MacLean DS, Robertson JD, Moody E, Stalker DJ, Jay M. Secondary $\mathrm{X}$-ray fluorescence for in vivo transdermal absorption measurements. Int J Pharm. 1996. (131):243-6.

7. O'Meara JM, Chettle DR, McNeill FE, Webber CE. In vivo X-ray fluorescence (XRF) measurement of uranium in bone. Appl Radiat Isot. 1998;49(5-6):713-5.

8. O'Meara JM, Börjesson J, Chettle DR. Improving the in vivo X-ray fluorescence (XRF) measurement of renal mercury. Appl Radiat Isot. 2000;53(4-5):639-46.

9. Bradley DA, Farquharson MJ. X-ray fluorescence and the in vivo evaluation of $\mathrm{Fe}, \mathrm{Cu}$ and $\mathrm{Zn}$ in skin. J Radioanal Nucl Chem. $2000 ;(244): 213-7$
10. O'Meara JM, Börjesson J, Chettle DR, McNeill FE. Optimization of an in vivo X-ray fluorescence mercury measurement system. Nucl. Instrum. Methods Phys. Res., Sect. B. 2004;(213):560-3.

11. Ali PA, Al-Hussany AF, Bennett CA, Hancock DA, El-Sharkawi AM. Plane polarized $\mathrm{x}$-ray fluorescence system for the in vivo measurement of platinum in head and neck tumours. Phys Med Biol. 1998;43(8):2337-45.

12. Shakeshaft J, Lillicrap SC. Technical note: an X-ray fluorescence system for the determination of gold in vivo following chrysotherapy. Br J Radiol. 1993;66(788):714-7.

13. Börjesson J, Barregård L, Sällsten G, Schütz A, Jonson R, Alpsten $\mathrm{M}$, et al. In vivo XRF analysis of mercury: the relation between concentrations in the kidney and the urine. Phys Med Biol. 1995; 40(3):413-26.

14. Christoffersson JO, Mattsson S. Polarised X-rays in XRF-analysis for improved in vivo detectability of cadmium in man. Phys Med Biol. 1983;28(10):1135-44.

15. Christoffersson JO, Welinder H, Spång G, Mattsson S, Skerfving S. Cadmium concentration in the kidney cortex of occupationally exposed workers measured in vivo using X-ray fluorescence analysis. Environ Res. 1987;42(2):489-99.

16. Al-Ghorabie FH, Natto SS, Al-Lyhiani SH. A comparison between EGS4 and MCNP computer modeling of an in vivo X-ray fluorescence system. Comput Biol Med. 2001;31(2):73-83.

17. Börjesson J, Mattsson S, Alpsten M. Trace element concentrations studied in vivo using X-ray fluorescence analysis. Appl. Radiat. Isot. 1998;(49):437-45.

18. Andrews NC. Disorders of iron metabolism. N Engl J Med. 1999;341(26):1986-95.

19. Michaëlsson G, Ljunghall K, Danielson BG. Zinc in epidermis and dermis in healthy subjects. Acta Derm Venereol. 1980;60 (4):295-9

20. Molin L, Wester PO. Iron content in normal and psoriatic epidermis. Acta Derm Venereol. 1973;53(6):473-6.

21. Brittenham GM, Griffith PM, Nienhuis AW, McLaren CE, Young NS, Tucker EE, et al. Efficacy of deferoxamine in preventing complications of iron overload in patients with thalassemia major. N Engl J Med. 1994;331(9):567-73.

22. Olivieri NF, Nathan DG, MacMillan JH, Wayne AS, Liu PP, McGee A, et al. Survival in medically treated patients with homozygous beta-thalassemia. N Engl J Med. 1994;331(9):574-8.

23. The Thalassemia Syndromes: Weatherall DJ, Clegg JB eds. Oxford, UK, Blackwell Scientific Publications, 1981.

24. Kremastinos DT, Tsetsos GA, Tsiapras DP, Karavolias GK, Ladis VA, Kattamis CA. Heart failure in beta thalassemia: a 5-year followup study. Am J Med. 2001;111(5):349-54.

25. Davis BA, Porter JB. Long-term outcome of continuous 24-hour deferoxamine infusion via indwelling intravenous catheters in highrisk beta-thalassemia. Blood. 2000;95(4):1229-36.

26. Gottschalk R, Wigand R, Dietrich CF, Oremek G, Liebisch F, Hoelzer D, Kaltwasser JP. Total iron-binding capacity and serum transferrin determination under the influence of several clinical conditions. Clin Chim Acta. 2000;293(1-2):127-38.

27. de Paula EV, Saad STO, Costa FF. Quelação oral de ferro na BetaTalassemia. Rev. bras. hematol. hemoter. 2003;1):59-63.

28. Trousseau A. Glycosurie, diabete sucre. In: Anonymous clinique medicale de l'Hotel-Dieu de Paris. 2.ed. Paris: Bailliere. 1865. p.663.

29. von Recklinghausen FD. Uber Hemochromatose. Tagebl Versamml Natur Arzte Heidelberg. 1889;(62):324.

30. Powell LW. Genetic diagnosis of hemochromatosis: implications for prophylaxis and treatment. In: Arroyo V, Bosch J, Bruguera 
M, editors. Therapy in liver diseases. Barcelona: Masson. 1997. p.391-404.

31. Galizzi Filho J, Andrade MO. Doença de Wilson e hemocromatose. In: Parise ER, Porta G, editores. Manual de diagnóstico e tratamento das doenças hepáticas - no paciente adulto e pediátrico. São Paulo: 1999. p.145-57.

32. Powell LW, Leggett BA, Crawford DHG. Hemochromatosis and other iron storage disorders. In: Schiff ER, Sorrell MF, Maddrey WC, editors. Schiff's diseases of the liver. $8^{\text {a }}$ ed. Philadelphia: Lippincott/Williams \& Wilkins. 1999. v.2, p.1107-30.

33. Powell LW, Subramaniam N, Yapp TR. Haemochromatosis in the new millennium.J Hepatol. 2000;32(1 Suppl):48-62. Review

34. de Souza AF, Carvalho-Filho RJ, Chebli JF. Hereditary hemochromatosis. Case report and review of the literature. Arq Gastroenterol. 2001;38(3):194-202.

35. Bothwell TH, MacPhail AP. Hereditary hemochromatosis: etiologic, pathologic, and clinical aspects. Semin Hematol. 1998;35(1): 55-71.

36. Brissot P, Moirand R, Guyader D, Loréal O, Turlin B, Deugnier Y. Hemochromatosis after the gene discovery: revisiting the diagnostic strategy. J Hepatol. 1998;28(Suppl 1):14-8.

37. Fargion S, Mandelli C, Piperno A, Cesana B, Fracanzani AL, Fraquelli M, et al. Survival and prognostic factors in 212 Italian patients with genetic hemochromatosis. Hepatology. 1992;15(4):655-9.

38. Turner JE. Atoms, Radiation, and Radiation Protection. 2. ed. New York. J. Wiley \& Sons, INC. 1995. cap 12 p. 366-373.

39. Berger MJ, Hubbell JH. XCOM: photon cross section on a personal computer.1987. NSBIR 87- 3597.

40. Aragão PHA, Suguihiro NM, Dos Santos SB, Estevam M, Da Costa ECS, Di Mauro E, et al. A Study of the elements in sooty mould phytopathology by physical techniques. Microsc Microanal. 2003. (9):1538-9

41. Aragão PH, dos Santos SB, da Costa EC, Suguihiro NM, Estevam M, Filho VF. Study of heavy metal content in HIV-infected blood using X-ray fluorescence technique. Cell Mol Biol (Noisy-le-grand). 2003;49 Online Pub:OL415-7.

42. Estevam M, Appoloni, CR. In vivo evaluation of Fe in human skin employing X-Ray Fluorescence Methodology (XRF). VI Latin American Symposium on Nuclear Physics and Applications. AIP Conf. Proc. 2007;(884):468-70.

Avaliação: Editor e dois revisores externos

Suporte Financeiro: Capes, Cnpq, Fundação Araucária, ProPPG- UEL.

Recebido: 04/06/2008

Aceito após modificações: 25/11/2008 